\title{
PREDIÇÃO E MODELAGEM DO EQUILÍBRIO LÍQUIDO- LÍQUIDO APLICADO À SEPARAÇÃO DE ÁCIDOS GRAXOS LIVRES NA PRODUÇÃO DE BIODIESEL
}

\author{
A. de A. ALBUQUERQUE ${ }^{1}$, L. DANIELSKI ${ }^{1}$ e L. STRAGEVITCH ${ }^{1}$ \\ ${ }^{1}$ Universidade Federal de Pernambuco, Departamento de Engenharia Química \\ E-mail para contato: allan-albuquerque123@hotmail.com
}

\begin{abstract}
RESUMO - Matérias-primas com alto teor de ácidos graxos livres (AGLs) para a produção de biodiesel são alternativas interessantes para a redução de custos. Convencionalmente, essas matérias-primas são processadas por uma etapa de esterificação seguida da transesterificação. Todavia, processos alternativos podem ser economicamente mais interessantes, como no caso da separação dos AGLs do óleo. Nesse caso, os AGLs são esterificados separadamente do óleo que é submetido à transesterificação. A avaliação desses processos alternativos requer o estudo do equilíbrio líquido-líquido de sistemas compostos de óleos vegetais/AGLs/metanol. Neste trabalho foi realizada uma avaliação do método UNIFAC para esses sistemas contendo metanol. Visando uma representação mais rigorosa do equilíbrio de fases necessário para a simulação e projeto de uma unidade de separação, também foi proposta uma modelagem empregando NRTL e UNIQUAC, aplicando para este último uma metodologia para reduzir o número de parâmetros ajustáveis. Foram usados dados experimentais disponíveis na literatura. Desvios consideráveis foram encontrados com o emprego do UNIFAC original. Parâmetros UNIFAC revisados existentes na literatura melhoram as predições, mas ainda não são satisfatórios para uma representação mais rigorosa do equilíbrio de fases. Por outro lado, NRTL e UNIQUAC possibilitaram obter uma representação mais realista dos dados experimentais, o que permitiria maior segurança nas etapas de projeto e simulação.
\end{abstract}

\section{INTRODUÇÃO}

Matérias-primas com alto teor de ácidos graxos livres (AGLs) para a produção de biodiesel são alternativas interessantes para a redução de custos. Tradicionalmente, estas são processadas por uma etapa de esterificação seguida de um estágio de transesterificação (Gnanaprakasam et al., 2013). A unidade de pré-tratamento aumenta o custo de capital investido em equipamentos, além do custo adicional com solvente, de forma que reduz a viabilidade econômica deste processo convencional relativamente ao uso de matérias-primas nobres mediante a reação de transesterificação (Zhang et al., 2003).

Processos alternativos visando a redução de custos podem ser concebidos pela separação dos AGLs dos triacilgliceróis (TAGs), a fim de aplicar separadamente as reações de esterificação e 


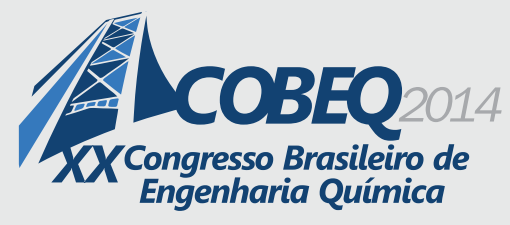

19 a 22 de outubro de 2014

Florianópolis/SC

transesterificação, respectivamente, a estas frações. Nesse caso, há a necessidade de estudar a separação de AGLs contidos em óleos empregados na produção de biodiesel. Esse estudo auxiliará no posterior dimensionamento e simulação de uma planta de produção de biodiesel com emprego de um método alternativo a partir de óleos e gorduras residuais (OGRs), de modo a realizar a comparação mediante avaliação técnica e econômica com o método convencional aplicado industrialmente. Dentre os métodos de separação possíveis, o emprego da extração líquido-líquido com um álcool de cadeia curta se apresenta como um processo promissor. Logo, para qualquer avaliação há a necessidade de dados de equilíbrio líquido-líquido (ELL) bem como uma modelagem adequada para sua representação rigorosa.

Neste trabalho foi realizada uma avaliação do método UNIFAC (Universal Quasi-Chemical Functional-Group Activity Coefficient) para sistemas compostos de óleos vegetais/AGLs/metanol aplicados à produção de biodiesel pela rota metílica. Visando uma representação mais rigorosa do equilíbrio de fases necessário para o projeto e a simulação de uma unidade de separação, também foi elaborada uma modelagem termodinâmica para esses sistemas empregando os modelos NRTL (NonRandom Two-Liquid) e UNIQUAC (Universal Quasi-Chemical). No caso do modelo UNIQUAC, foi usada a metodologia proposta por Poling et al. (2001) para reduzir o número de parâmetros de interação ajustáveis entre TAGs.

\section{METODOLOGIA}

Para realizar a predição e a modelagem do ELL de sistemas compostos de óleos vegetais/AGLs/álcool aplicados a produção de biodiesel foram considerados apenas dados contendo metanol como álcool. O biodiesel produzido pela rota metílica em geral apresenta maiores rendimentos que outros de cadeias carbônicas mais longas. Segundo Ma e Hanna (1999), o seu uso é mais frequente devido seu baixo custo e suas vantagens físico-químicas. Assim, o próprio metanol empregado na separação servirá para ambas as reações de esterificação e transesterificação.

Dados de ELL envolvendo sistemas do tipo óleos vegetais/AGLs/metanol foram reportados na literatura por Batista et al. (1999 a), Mohsen-Nia e Dargahi (2007) e Mohsen-Nia e Khodayari (2008). Todavia, na predição e na modelagem realizadas com UNIFAC e NRTL, somente foram considerados os sistemas de ELL de óleo de milho (OM)/ácido oleico (AO)/metanol (M) e óleo de girassol $(\mathrm{OG}) / \mathrm{AO} / \mathrm{M}$ ambos a $30^{\circ} \mathrm{C}$ e a $40^{\circ} \mathrm{C}$. Tais dados estudados pelos dois últimos grupos de autores foram selecionados pois não foram explorados com estes modelos, além de possuírem seus principais TAGs constituintes semelhantes. A Tabela 1 apresenta a provável composição de TAGs dos óleos de girassol e de milho determinada através da metodologia de Antoniosi Filho et al. (1995). Nesse método as composições são baseadas nos componentes de maiores concentrações no conjunto de isômeros com x carbonos e y ligações duplas. Por isso alguns TAGs presentes nas composições dadas por Mohsen-Nia e Dargahi (2007) e Mohsen-Nia e Khodayari (2008) não aparecem explicitamente na Tabela 1. Como exemplo, tem-se o SLnL que não se apresenta explicitamente na Tabela 1, mas faz parte do grupo 54:5 com o principal TAG sendo o OLL. Vale ressaltar que foram desconsiderados os componentes com menos de $0,4 \%$ e $0,8 \%$ para os óleos de girassol e de milho, respectivamente, a fim de manter um máximo de dez TAGs como representativos para cada óleo. Além disso, na predição e na modelagem foram considerados os dados de ELL em base mássica devido a grande diferença entre 
as massas molares dos componentes dos sistemas, seguindo Oishi e Prausnitz (1978).

Tabela 1 - Provável composição de TAGs do óleo de girassol e de milho iranianos

\begin{tabular}{ccccc}
\hline & & & \multicolumn{2}{c}{ Fração em massa (\%) } \\
Grupo & TAG principal & $\mathrm{MM}^{\mathrm{b}}\left({\left.\mathrm{g} \cdot \mathrm{mol}^{-1}\right)}^{\mathrm{n}}\right)$ & Óleo de girassol & Óleo de milho \\
\hline $50: 1^{\mathrm{a}}$ & POP & 833,37 & - & 0,87 \\
$50: 2$ & PLP & 831,35 & 1,05 & 1,82 \\
$52: 2$ & POO & 859,40 & 2,03 & 3,25 \\
$52: 3$ & PLO & 857,39 & 6,68 & 10,12 \\
$52: 4$ & PLL & 857,39 & 9,63 & 10,74 \\
$54: 2$ & SOO & 855,38 & 0,75 & 0,88 \\
$54: 3$ & SLO & 885,44 & 4,02 & 4,67 \\
$54: 4$ & OLO & 883,43 & 14,68 & 16,52 \\
$54: 5$ & OLL & 881,41 & 30,75 & 29,73 \\
$54: 6$ & LLL & 879,40 & 29,74 & 21,40 \\
$54: 7$ & LLnL & 877,38 & 0,67 & - \\
& Total & & 100 & 100 \\
\hline
\end{tabular}

${ }^{\mathrm{a}} \mathrm{x}: \mathrm{y}, \mathrm{x}=$ número de carbonos (exceto carbonos do glicerol) e y = número de ligações duplas.

${ }^{\mathrm{b}} \mathrm{MM}=$ Massa molar.

Para realizar a comparação entre os valores experimentais e calculados foi empregado o desvio médio quadrático $(\Delta w)$ de acordo com a Equação 1. Os sobrescritos $I$ e II representam as fases, enquanto $c a l$ e $\exp$ são calculado e experimental, respectivamente. $N$ e $C$ são os números de linhas de amarração e de componentes, respectivamente, e $w$ é a fração mássica.

$$
\Delta w=100 \sqrt{\frac{\sum_{j=1}^{N} \sum_{i=1}^{C}\left[\left(w_{i j}^{I, \text { exp }}-w_{i j}^{I, c a l}\right)^{2}+\left(w_{i j}^{I I, e x p}-w_{i j}^{I I, c a l}\right)^{2}\right]}{2 N C}}
$$

\subsection{Predição dos dados de ELL}

A predição dos dados de ELL dos sistemas compostos de OG/AO/M e OM/AO/M, ambos a $30^{\circ} \mathrm{C}$ e a $40^{\circ} \mathrm{C}$, foi realizada com o método UNIFAC empregando os parâmetros dados por Magnussen et al. (1981) e Batista et al. (1999 b), sendo que este último originalmente foi ajustado para sistemas etílicos. Foram consideradas as composições dadas na Tabela 1 para representação dos óleos. Após a realização do cálculo flash multicomponente, as frações dos TAGs contidos nos óleos foram agrupadas e comparadas aos valores experimentais mediante o $\Delta w$ dado pela Equação 1 . 


\subsection{Modelagem termodinâmica}

Para os mesmos dados foi também realizada a modelagem termodinâmica com os modelos NRTL e UNIQUAC. Neste último também foram incluídos os dados de Batista et al. (1999 a) para o sistema óleo de canola(OC)/AO/M a $30^{\circ} \mathrm{C}$. Os parâmetros dos modelos foram obtidos seguindo a metodologia proposta por Stragevitch e d'Ávila (1997). Nessa é empregado o método simplex para minimizar a função objetivo à base de concentração conforme a Equação 2,

$$
F=\sum_{k}^{D} \sum_{j}^{N} \sum_{i}^{C-1}\left[\left(\frac{w_{i j k}^{I, \text { calc }}-w_{i j k}^{I, \text { exp }}}{\sigma_{w_{i j k}^{I}}}\right)^{2}+\left(\frac{w_{i j k}^{I I, \text { calc }}-w_{i j k}^{I I, \exp }}{\sigma_{w_{i j k}^{I I}}^{I I}}\right)^{2}\right]
$$

onde $D$ representa o número de conjuntos de dados, $\sigma_{w_{i j k}^{I}}$ e $\sigma_{w_{i j k}^{I I}}$ são as incertezas observadas em $w$ para ambas as fases líquidas. O restante dos subscritos e sobrescritos são os mesmos da Equação 1.

O modelo NRTL foi usado considerando o sistema como pseudoternário com OG e OM como pseudocomponentes. As frações mássicas calculadas e experimentais foram comparadas pela Equação 1. Para reduzir a quantidade de parâmetros ajustáveis do modelo UNIQUAC foi adotada a metodologia de Poling et al. (2001) para estimar os parâmetros de interação dos TAGs presentes no óleo, de acordo com uso das Equações 3, 4 e 5,

$$
\begin{aligned}
& \Delta U_{i} \cong \Delta H_{v i}-R T \\
& u_{i i}=\frac{-\Delta U_{i}}{q_{i}} \\
& u_{i j}=u_{j i}=\left(u_{i i} u_{j j}\right)^{\frac{1}{2}}\left(1-c_{i j}\right)
\end{aligned}
$$

onde $\Delta U_{i}$ e $\Delta H_{v i}$ são, respectivamente, a energia interna e a entalpia de vaporização, longe da temperatura crítica, $R$ é a constante universal dos gases, $q_{i}$ é o parâmetro UNIFAC de área e $u_{i j}$ é o parâmetro de interação entre os componentes $i$ e $j, c_{i j}$ é um parâmetro binário ajustável que para misturas de líquidos apolares é positivo e menor comparado com a unidade. Logo, $c_{i j}$ foi considerado nulo visto que os TAGs possuem cadeias carbônicas apolares extensas.

Para a representação dos sistemas de óleos de girassol e de milho foram considerados os três TAGs principais que são os mesmos, conforme a Tabela 1, ou seja, OLL, LLL e OLO. Esses foram normalizados e considerados por hipótese que sua distribuição é a mesma em ambas as fases, o que possibilitou a regressão dos dados conjuntamente em busca dos parâmetros de interação. Assim, foram computadas as interações entre os três TAGs a partir das equações acima e mantidas fixas durante a correlação dos dados de ELL. Além disso, devido a não disponibilidade de dados 
experimentais de $\Delta H_{v i}$ dos TAGs, esses foram preditos pela metodologia de Zong et al. (2010). Com essas considerações, também foram incluídos na regressão os dados de ELL reportados por Batista et al. (1999 a) a $30^{\circ} \mathrm{C}$ considerando os três TAGs principais OLO, OLL e OOO, conforme normalização da provável composição de TAGs apresentada naquele trabalho. Para todos os componentes inclusos anteriormente foram mantidos fixos os seus parâmetros de interação, além dos calculados entre OOO com OLO e OLL. Assim, foram ajustados apenas os parâmetros relativos à OOO com ácido oleico e metanol. Após o cálculo flash multicomponente, as frações mássicas dos TAGs contidos nos óleos foram agrupadas e comparadas aos valores experimentais mediante o $\Delta w$ dado pela Equação 1 .

\section{RESULTADOS E DISCUSSÃO}

\subsection{Predição do ELL}

A Figura 1 mostra a comparação dos dados de ELL experimentais e preditos pelo método UNIFAC, para o sistema de $\mathrm{OG} / \mathrm{AO} / \mathrm{M}$ a $30^{\circ} \mathrm{C}$ com os parâmetros dados por (a) Magnussen et al. (1981) e (b) Batista et al. (1999 b).

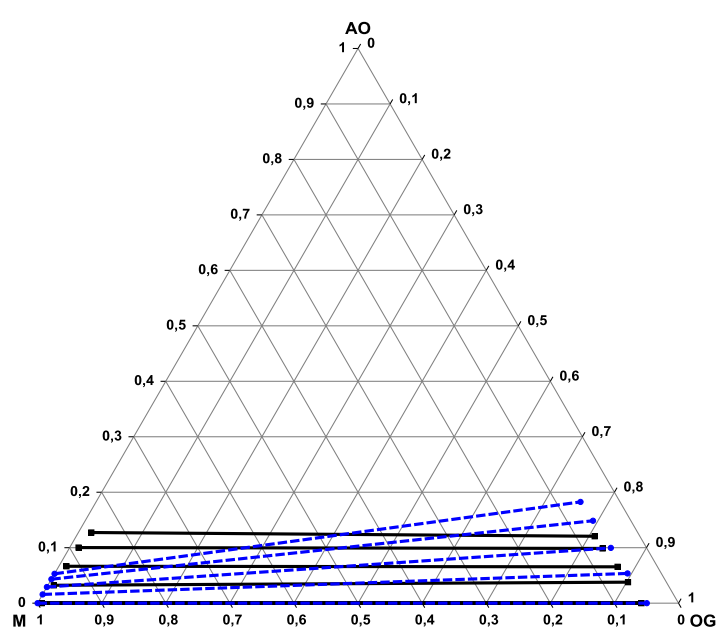

(a)

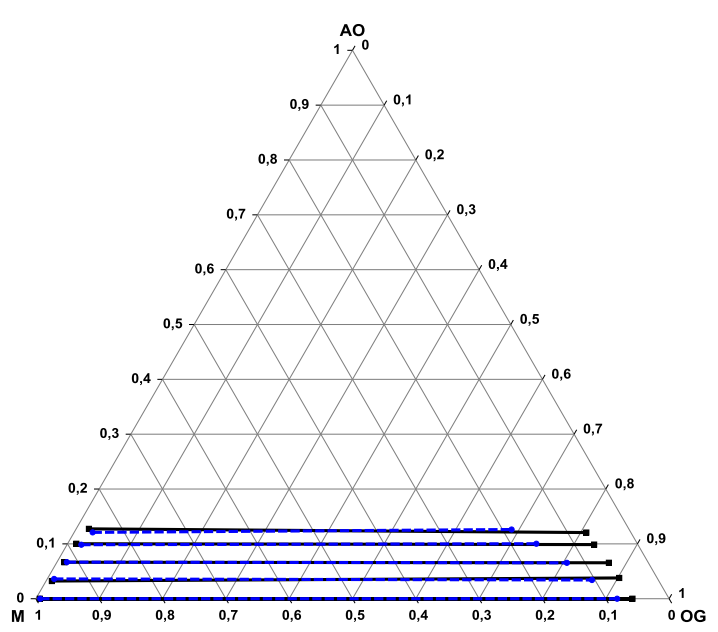

(b)

Figura 1 - Comparação dos dados de ELL experimentais e preditos para o sistema $\mathrm{OG} / \mathrm{AO} / \mathrm{M}$ a $30^{\circ} \mathrm{C}$ pelo método UNIFAC com os parâmetros de (a) Magnussen et al. (1981) e (b) Batista et al. (1999 b):

(匹) experimental (Mohsen-Nia e Khodayari, 2008); (-•-) UNIFAC.

Como pode ser observado, o uso da tabela de parâmetros de Magnussen et al. (1981) previu incorretamente a inclinação das tie lines. Isso demonstra uma previsão insatisfatória dos coeficientes de distribuição que teriam grande influência no projeto e na simulação dos equipamentos de extração. Com o emprego dos parâmetros revisados por Batista et al. (1999 b) foram obtidas inclinações corretas, porém, foi calculada uma solubilidade maior da fase álcool na fase rica em óleo do que aquela observada experimentalmente, o que pode ser resultado da extrapolação desses parâmetros para o sistema metílico. Resultados semelhantes foram obtidos para o sistema OM/AO/M, porém valores menores do $\Delta w$ foram encontrados com os parâmetros revisados por Batista et al. (1999 b). 


\subsection{Modelagem termodinâmica}

A Figura 2 mostra a comparação dos dados de ELL experimentais e calculados para o sistema de $\mathrm{OG} / \mathrm{AO} / \mathrm{M}$ a $40^{\circ} \mathrm{C}$ pelo modelo (a) NRTL e (b) UNIQUAC.

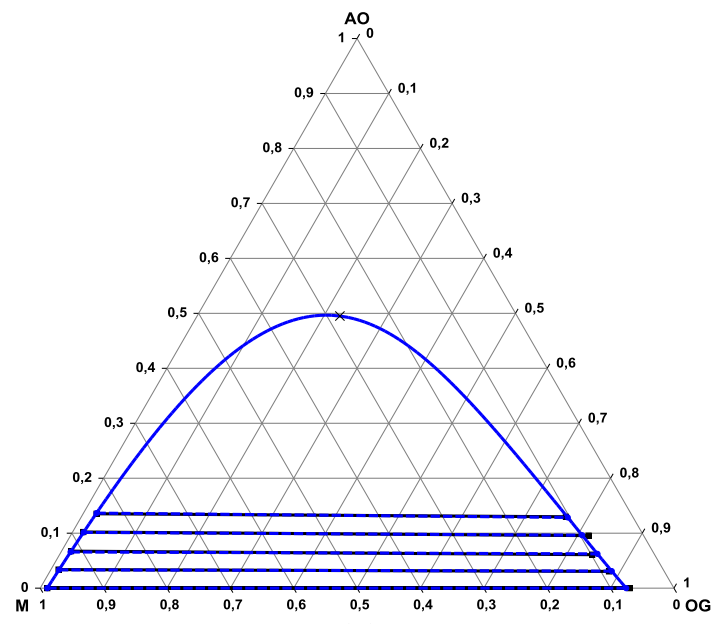

(a)

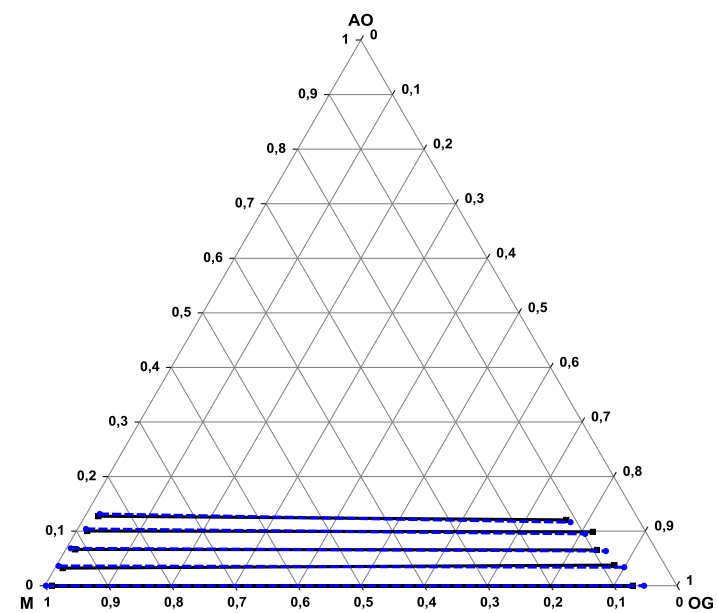

(b)

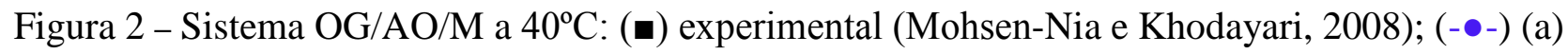
NRTL e (b) UNIQUAC; $(\times)$ plait point.

Com o modelo NRTL o sistema de OG apresentou desvios menores quando considerados parâmetros específicos para cada temperatura, o que demonstra certa dependência desses com a temperatura. No caso do sistema de OM, por outro lado, um mesmo conjunto de parâmetros foi suficiente para ambas as temperaturas. Para UNIQUAC a abordagem de redução do número de parâmetros ajustáveis foi satisfatória para uma boa representação dos dados experimentais de OG e de OM. Resultados semelhantes foram obtidos para o sistema $\mathrm{OC} / \mathrm{AO} / \mathrm{M}$ a $30^{\circ} \mathrm{C}$ considerando os TAGs OLO, OLL e OOO como representativos após normalização. Logo, o emprego destes modelos possibilitaria maior segurança nas etapas de projeto e simulação do extrator da unidade de separação.

As Tabelas 2 e 3 mostram os parâmetros NRTL e UNIQUAC, respectivamente, obtidos para os sistemas considerados. A Tabela 4 apresenta o $\Delta w$ das composições mássicas das fases. Conforme observado, os modelos NRTL e UNIQUAC são os preferíveis para a representação dos sistemas estudados, apresentando desvios médios menores do que as respectivas predições usando UNIFAC.

Tabela 2 - Parâmetros NRTL do sistema OG ou OM (1)/AO (2)/M (3)

\begin{tabular}{cccccccccc}
\hline \multirow{2}{*}{$\mathrm{ij}$} & \multicolumn{3}{c}{$\mathrm{OG} / \mathrm{AO} / \mathrm{M}$ a $30^{\circ} \mathrm{C}$} & \multicolumn{3}{c}{$\mathrm{OG} / \mathrm{AO} / \mathrm{M}$ a $40^{\circ} \mathrm{C}$} & \multicolumn{3}{c}{$\mathrm{OM} / \mathrm{AO} / \mathrm{M}$} \\
& $\mathrm{A}_{\mathrm{ij}}(\mathrm{K})$ & $\mathrm{A}_{\mathrm{ji}}(\mathrm{K})$ & $\alpha_{\mathrm{ij}}$ & $\mathrm{A}_{\mathrm{ij}}(\mathrm{K})$ & $\mathrm{A}_{\mathrm{ji}}(\mathrm{K})$ & $\alpha_{\mathrm{ij}}$ & $\mathrm{A}_{\mathrm{ij}}(\mathrm{K})$ & $\mathrm{A}_{\mathrm{ji}}(\mathrm{K})$ & $\alpha_{\mathrm{ij}}$ \\
\hline 12 & $-15,55$ & $-114,93$ & 0,200 & 2987,6 & 0,18 & 0,470 & 177,21 & 23,67 & 0,470 \\
13 & $-313,96$ & 2519 & 0,258 & $-769,66$ & 3104,3 & 0,200 & 98,88 & 2141,2 & 0,339 \\
23 & 3615,8 & 408,38 & 0,200 & 790,6 & 425,81 & 0,225 & $-1148,6$ & 2334,7 & 0,233 \\
\hline
\end{tabular}


Tabela 3 - Parâmetros de interação UNIQUAC válidos para os sistemas de óleos considerados: OLL (1)/LLL (2)/ OLO (3)/ OOO (4)/AO (5)/M (6)

\begin{tabular}{ccclccccc}
\hline $\mathrm{ij}$ & $\mathrm{A}_{\mathrm{ij}}(\mathrm{K})$ & $\mathrm{A}_{\mathrm{ji}}(\mathrm{K})$ & $\mathrm{ij}$ & $\mathrm{A}_{\mathrm{ij}}(\mathrm{K})$ & $\mathrm{A}_{\mathrm{ji}}(\mathrm{K})$ & $\mathrm{ij}$ & $\mathrm{A}_{\mathrm{ij}}(\mathrm{K})$ & $\mathrm{A}_{\mathrm{ji}}(\mathrm{K})$ \\
\hline $12^{\mathrm{a}, \mathrm{b}}$ & 2,1914 & $-2,184$ & $23^{\mathrm{a}, \mathrm{b}}$ & $-4,3311$ & 4,3606 & 35 & $-0,0964$ & $-22,161$ \\
$13^{\mathrm{a}, \mathrm{b}}$ & $-2,1545$ & 2,1618 & $24^{\mathrm{a}, \mathrm{c}}$ & $-6,4422$ & 6,508 & 36 & 171,74 & 26,073 \\
$14^{\mathrm{a}, \mathrm{b}}$ & $-4,2729$ & 4,3019 & 25 & 68,914 & $-98,161$ & 45 & $-0,2842$ & 8,7469 \\
15 & 60,051 & 113,65 & 26 & 279,91 & 55,849 & 46 & 134,77 & 41,344 \\
16 & 354,21 & $-34,759$ & $34^{\mathrm{a}, \mathrm{b}}$ & $-2,1257$ & 2,1328 & 56 & $-135,98$ & 290,83 \\
\hline
\end{tabular}

${ }^{\mathrm{a}}$ Obtidos pela metodologia de Poling et al. (2001). ${ }^{\mathrm{b}}$ Mantidos fixos na regressão dos dados de ELL. ${ }^{c}$ Não empregado no ajuste devido não haver a interação LLL com OOO.

Tabela 4 - Desvio médio quadrático das composições mássicas das fases

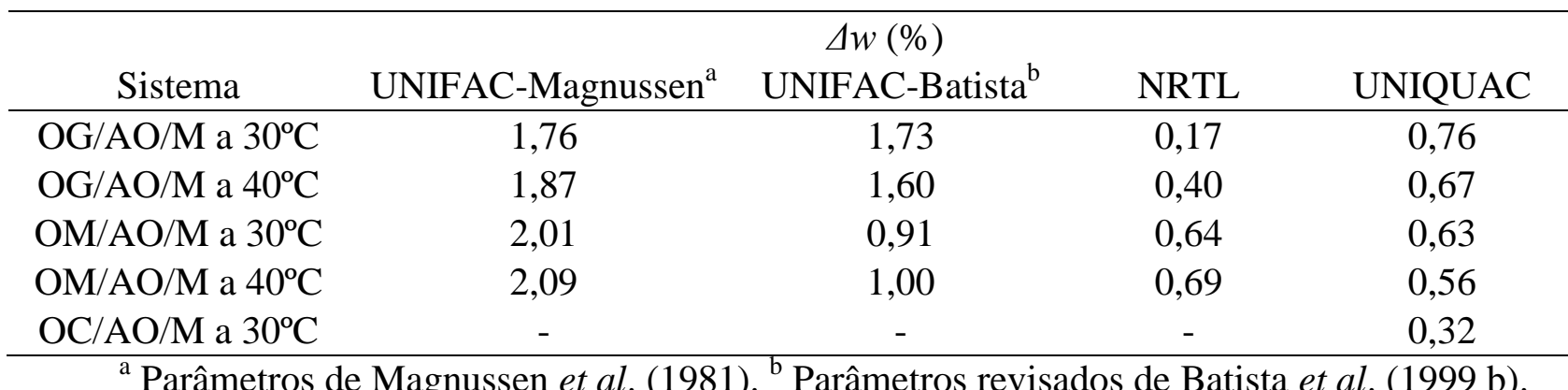

\section{Conclusões}

Para a predição dos dados de ELL com UNIFAC foram empregados os parâmetros de Magnussen et al. (1981) e os parâmetros revisados por Batista et al. (1999 b). Com a tabela de Magnussen et al. (1981) foram obtidas inclinações das tie lines diferentes das experimentais. Para os parâmetros revisados de Batista et al. (1999 b) estendidos para os sistemas metílicos foi mostrado que, apesar da maior solubilidade da fase metanol na fase óleo, foram obtidas inclinações das tie lines próximas das experimentais.

Com a modelagem realizada usando NRTL e UNIQUAC foi obtida uma boa representação dos dados de ELL, apresentando baixos desvios médios quadráticos das composições calculadas. Estes confirmaram as expectativas da existência de diagramas do tipo I e apresentaram inclinações das tie lines próximas das experimentais. No caso do modelo UNIQUAC os sistemas foram representados com três TAGs e podem ser aplicados ao extrator com os quatro TAGs (OLL, LLL, OLO e OOO). No entanto, como possíveis limitações dessa abordagem empregada para uso geral com qualquer matéria prima graxa pode-se citar o reduzido número de TAGs usados. Além disso, a não disponibilidade de informações sobre a distribuição dos TAGs entre as fases também foi outra simplificação necessária. 


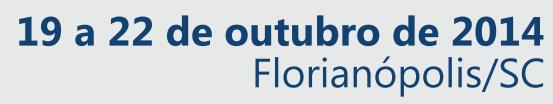

Portanto, os conjuntos de parâmetros estimados por NRTL e UNIQUAC podem ser úteis para o projeto e a simulação do extrator presente em um processo alternativo de produção de biodiesel a partir de OGRs. Sobretudo a modelagem proposta com UNIQUAC possibilitará uma melhor representação dessas matérias-primas em simuladores tais como o Aspen ${ }^{\circledR}$, pois, em geral, essas são formadas de misturas de vários óleos processados.

\section{REFERÊNCIAS}

ANTONIOSI FILHO, N. R.; MENDES, O. L.; LANÇAS, F. M. Computer Prediction of Triacylglycerol Composition of Vegetable Oils by HRGC. Chromatographia, v. 40, p. 557-562, 1995.

BATISTA, E. et al. Liquid-Liquid Equilibrium for Systems of Canola Oil, Oleic Acid, and ShortChain Alcohols. J. Chem. Eng. Data, v. 44, p. 1360-1364, 1999 a.

BATISTA, E. et al. Prediction of Liquid-Liquid Equilibrium for Systems of Vegetable Oils, Fatty Acids, and Ethanol. J. Chem. Eng. Data, v. 44, p. 1365-1369, 1999 b.

GNANAPRAKASAM, A. et al. Recent Strategy of Biodiesel Production from Waste Cooking Oil and Process Influencing Parameters: A Review. J. Energy, v. 2013, p. 1-11, 2013.

MA, F.; HANNA, M. A. Biodiesel production: a review, Bioresource Technol., v. 70, p. 1-15, 1999.

MAGNUSSEN, T.; RASMUSSEN, P.; FREDENSLUND, A. UNIFAC Introduction Parameter Table for Prediction of Liquid-Liquid Equilibria. Ind. Eng. Chem. Proc. DD., v. 20, p. 331-339, 1981.

MOHSEN-NIA, M.; DARGAHI, M. Liquid-Liquid Equilibrium for Systems of (Corn Oil + Oleic Acid + Methanol or Ethanol) at (303.15 and 313.15) K. J. Chem. Eng. Data, v. 52, p. 910-914, 2007.

MOHSEN-NIA, M.; KHODAYARI, A. De-acidification of sunflower oil by solvent extraction: (Liquid + liquid) equilibrium data at $\mathrm{T}=(303.15$ and 313.15) K. J. Chem. Thermodyn, v. 40, p. 1325-1329, 2008.

OISHI, T.; PRAUSNITZ, J. M. Estimation of Solvent Activities in Polymer Solutions Using a GroupContribution Methodology. Ind. Eng. Chem. Process DD., v. 17, p. 333-339, 1978.

POLING, B. E.; PRAUSNITZ, J. M.; O'CONNELL, J. P. The properties of gases and liquids. New York: Mcgraw-hill, 2001.

STRAGEVITCH, L.; D’ÁVILA, S.G. Application of a generalized maximum likelihood method in the reduction of multicomponent liquid-liquid equilibrium data. Braz. J. Chem. Eng., v. 14, p. 41-52. 1997.

ZHANG, Y. et al. Biodiesel production from waste cooking oil: 2. Economic assessment and sensitivity analysis. Bioresource Technol., v. 90, p. 229-240, 2003.

ZONG, L.; RAMANATHAN, S.; CHEN, C. Predicting Thermophysical Properties of Mono- and Diglycerides with the Chemical Constituent Fragment Approach. Ind. Eng. Chem. Res., v. 49, p. 5479-5484, 2010. 